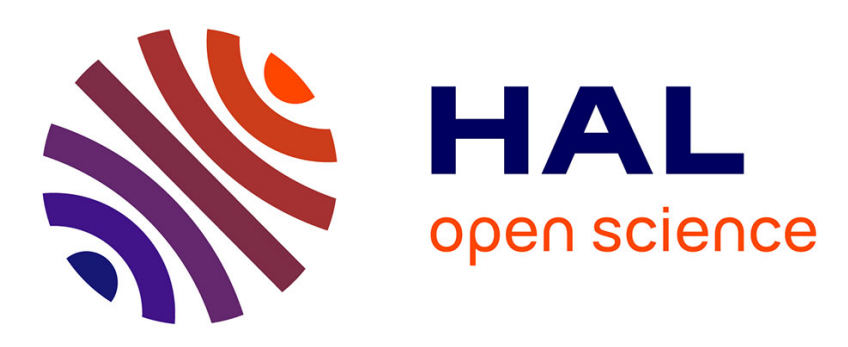

\title{
Interactive robotics for human impedance estimation in a rhythmic task
}

Vincent Fortineau, Maria Makarov, Pedro Rodriguez-Ayerbe, Isabelle Anne Siegler

\section{- To cite this version:}

Vincent Fortineau, Maria Makarov, Pedro Rodriguez-Ayerbe, Isabelle Anne Siegler. Interactive robotics for human impedance estimation in a rhythmic task. 2020 IEEE 16th International Conference on Automation Science and Engineering (CASE), Aug 2020, Hong-Kong, China. 10.1109/CASE48305.2020.9217009 . hal-02900405

\section{HAL Id: hal-02900405 \\ https://hal.science/hal-02900405}

Submitted on 22 Mar 2021

HAL is a multi-disciplinary open access archive for the deposit and dissemination of scientific research documents, whether they are published or not. The documents may come from teaching and research institutions in France or abroad, or from public or private research centers.
L'archive ouverte pluridisciplinaire HAL, est destinée au dépôt et à la diffusion de documents scientifiques de niveau recherche, publiés ou non, émanant des établissements d'enseignement et de recherche français ou étrangers, des laboratoires publics ou privés. 


\title{
Interactive robotics for human impedance estimation in a rhythmic task
}

\author{
Vincent FORTINEAU ${ }^{1,2}$, Maria MAKAROV ${ }^{1}$, Pedro RODRIGUEZ-AYERBE ${ }^{1}$, Isabelle A. SIEGLER ${ }^{2}$
}

\begin{abstract}
This paper presents an experimental robotic setup dedicated to human arm endpoint impedance measurement in a cyclic task with physical interactions. Understanding human endpoint impedance adaptation during a cyclic task in interaction with the environment can provide insights for the design of innovative robot controllers for collaborative robotics both in manufacturing and service contexts. The ball bouncing benchmark task, classical in human movement science, is selected here for its simplicity, yet representativeness of handeye synchronization and intermittent physical interactions. For a better control over the environmental conditions, the task is performed in a simulated environment, where the paddle is actuated by the human participant by interacting with an admittance-controlled collaborative robotic arm. First experimental feasibility and identification results are presented.
\end{abstract}

\section{INTRODUCTION}

In the longtime research topic of human motor control [1], recent developments benefit from computational approaches and concepts closely related to the automatic control field. The central nervous system (CNS) and the intrinsic biomechanical properties of humans limbs allow coordinated and efficient movements during interaction with their environment [2]. Especially, humans excel in physical interactions with environments with a wide range of physical properties, potentially unknown beforehand [1], [2]. Humans are capable of adapting the dynamic properties of their limbs [3] to increase performances during the learning of a new task [4], and reach stable and desired behaviour. Understanding and reproducing some properties of how humans interact with their environment during various tasks is thus relevant for the design of innovative robot control strategies.

The mechanical impedance of human limbs extends beyond stiffness and has been studied for decades under the control prism [7], and is still an active research topic [8]. The notion of impedance relates kinematics to forces, allowing the modeling of human movements during physical interactions. Very simple models, such as mass-spring-damper (KBI) or Hill type muscle models, have been shown to describe the human behaviour [6]. These models do not intended to explain the complete human motor control, but rather reproduce some of its aspects. More complete models [10], [21] are required to understand the human CNS.

Human movements can be separated in categories for better modelling. The differences between rhythmic and discrete movements on the side of human motor control were defined and discussed in [11]. This paper focuses on rhythmic tasks. Elbow joint kinematic and dynamic properties during cyclic

\footnotetext{
1 Laboratoire des signaux et systèmes, Université ParisSaclay, CNRS, CentraleSupélec, 91190, Gif-sur-Yvette, France vincent. fortineaudcentralesupelec.fr

${ }^{2}$ CIAMS, Université Paris-Saclay, 91405, Orsay, France, \& Université d'Orléans, 45067, Orléans, France
}

task without load were studied in [5]. They were able to connect the maximum frequency with the magnitude of the movement, and then with the arm stiffness, observing both stiffness and frequency increase together. They proposed a relation between the joint stiffness and the muscular activity, stating in corroboration with previous studies [7], that humans can intentionally control their arm stiffness notably thanks to the co-contraction of agonist and antagonist muscles.

On one hand, the studies on human motor control are essential for better interactions between humans and robots [9], and on another hand they inspire new robotic designs for cobots or to try to reproduce human performances. A bioinspired torque-stiffness control strategy for dynamic walking was proposed in [12], where central pattern generators (CPG) shape the variations of a robot joint stiffness. This approach was shown to improve the energy efficiency of the task compared to a torque control strategy alone.

In previous work at the intersection of robotics, control and human movement sciences [13], [14], the authors studied the human CNS during cyclic movements in a ball bouncing task in a purely kinematic simulated environment. They were able to reproduce human-like performances using a CPG-based control structure. The Matsuoka oscillator played the role of the CPG, taking the kinematic data of the ball as input to control both amplitude and frequency of the elbow joint angle of a simulated human arm.

This paper proposes a robotic experimental set-up to identify the human impedance by means of an interactive robot. The experience is designed to identify the human arm endpoint impedance variations during a cyclic task, requiring eye-hand coordination, i.e. the ball bouncing task. Compared to the previous works [13], [14] on the same task, the modeling objective thus goes beyond the kinematic level, and concentrates now on the interaction forces.

The rhythmic ball bouncing task and human impedance model are recalled in Section II. How interactive robotics may be used to identify human impedance is then detailed in Section III. The experimental implementation details are given in Section IV, and preliminary results using this implementation are presented in Section V.

\section{TASK DESCRIPTION AND IMPEDANCE ESTIMATION}

The modeling of the ball bouncing task and the assumptions regarding the human impedance are briefly recalled.

\section{A. The ball bouncing task}

1) Ball kinematics: The ball bouncing task is a well known task in Human Movement Science, in which subjects, often experts, are asked to bounce a ball to reach a target height $h$.The task is depicted on Fig. 1. Between impacts $k$ and 


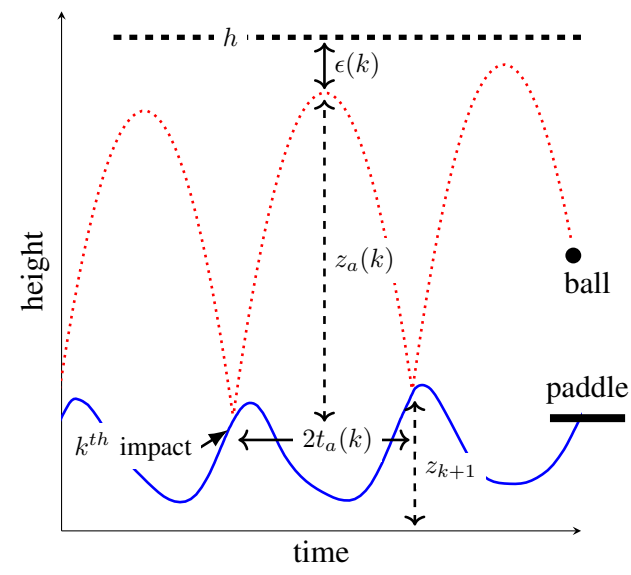

Fig. 1: Paddle (solid blue line) and ball (red dotted line) trajectories during the ball bouncing task with target height $h$ (black dashed line).

$k+1$ at times $t_{k}$ and $t_{k+1}$, the motion of the ball is described by the ballistic equation (1), with $z(t)$ the ball position, $\dot{z}_{k}^{+}$ the post-impact velocity of the ball at $t_{k}, z_{k}$ the ball impact position at $t_{k}, t$ the time elapsed since the last impact $k$ and $g$ the gravitational acceleration.

$$
z(t)=-0.5 g t^{2}+\dot{z}_{k}^{+} t+z_{k}
$$

The impact of the ball on the paddle is modeled by (2), using a restitution coefficient $\alpha$ in a simplified manner, as described in [13]. Paddle position is denoted $p, m_{b}$ and $m_{p}$ are respectively the ball and the paddle masses, and $\dot{z}_{k}^{-}=\dot{z}_{k-1}^{+}-g t$ is the velocity of the ball before the impact $k$.

$$
\dot{z}_{k}^{+}=\frac{m_{p}\left((1+\alpha) \dot{p}_{k}-\alpha \dot{z}_{k}^{-}\right)+m_{b} \dot{z}_{k}^{-}}{m_{p}+m_{b}}
$$

2) Ball bouncing feasibility domain: Several characteristic constants can be derived from the previous equations. The time of apex between impacts $k$ and $k+1$ is $t_{a}=\dot{z}_{k}^{+} / g$, and the frequency of the task $f=1 /\left(2 t_{a}\right)$, that is $f=$ $g /\left(2 \dot{z}_{k}^{+}\right)$, allowing to link the ball apex height $z_{a}$, the postimpact velocity and the frequency: $z_{a}-z_{k}=\left(\dot{z}_{k}^{+}\right)^{2} /(2 g)=$ $\dot{z}_{k}^{+} /(4 f)$.

Results found in [5] pointed out that for an amplitude of cyclic movements of the elbow joint, around $1.25 \mathrm{rad}$, the maximum frequency was $2 \mathrm{~Hz}$, growing up until $8.4 \mathrm{~Hz}$ for almost null amplitudes. Since the ball bouncing task requires an active synchronization between the movements of both the arm and ball, smaller frequencies and magnitudes should be considered to avoid designing an almost impossible task, or a task requiring complex arm motions. However, the task frequency should be high enough to yield a rhythmic behaviour. The overall objective being to reproduce this rhythmic task using interactive robotics, feasible conditions can be deduced from the previous equations and post-impact velocities similar to those considered in [27].

\section{B. Human impedance in a rhythmic task}

In a cyclic task performed by a human arm, its endpoint impedance defines the dynamic behavior of the arm when it is deviated by an external perturbation from the nominal trajectory which it would follow without the perturbation, called virtual trajectory in [15]. The KBI model (3) for the mechanical impedance relating forces to motion is considered:

$$
\delta \boldsymbol{f}=\boldsymbol{I} \delta \ddot{\boldsymbol{x}}+\boldsymbol{B} \delta \dot{\boldsymbol{x}}+\boldsymbol{K} \delta \boldsymbol{x},
$$

where $\delta \boldsymbol{x}$ is the difference between the virtual and the perturbed trajectory of the human hand and $\delta \boldsymbol{f}$ the difference between the virtual and perturbed interaction force. $K, B$ and $\boldsymbol{I}$ are respectively the stiffness (in $\mathrm{N} \mathrm{m}^{-1}$ ), damping (in $\mathrm{N} \mathrm{s} \mathrm{m}^{-1}$ ) and mass (in $\mathrm{kg}$ ) $\mathbb{R}^{n \times n}$ matrices, $n$ being the number of Cartesian DOF.

The introduction of perturbations is thus necessary for impedance identification. Perturbations can have various durations, but for a task in movement, they usually last few hundreds of milliseconds [16], [22], [26], and can be injected either as forces/torques or positions perturbations. The magnitude of the perturbations remains low, since most common impedance models of human limbs are linearizations [23], that hold for small movements $\delta \boldsymbol{x}$. Typical force perturbations range around $4 \mathrm{~N}$ [22], [25], or for position perturbations, below $1 \mathrm{~cm} \mathrm{[16].}$

This paper does not intend to differentiate the contribution of reflexes from intrinsic properties of the human arm, like it was done in [21], but rather try to observe the global behaviour of the arm. To that purpose, the simplified KBI model (3) for the human arm behaviour is proposed at the endpoint level, without coupling between the different axes, that is with diagonal parameter matrices. Considering that the ball bouncing task is reduced to the vertical $\boldsymbol{z}$-axis, the identified impedance reduces here to a single degree of freedom:

$$
\delta f_{z}=I_{z} \delta \ddot{x}_{z}+B_{z} \delta \dot{x}_{z}+K_{z} \delta x_{z}
$$

Moreover, since this model cannot account for voluntary action, the latter should be discriminated by experimental design and data post-treatment (see Section V-B).

\section{INTERACTIVE ROBOTICS AS A TOOL FOR HUMAN IMPEDANCE ESTIMATION}

This section presents the proposed methodology for the use of interactive robotics to help the analysis of human impedance during rhythmic tasks.

\section{A. Methodology}

The ball bouncing task takes place in a simulated environment in which experimental conditions (gravity, restitution coefficient...) are easier to control. The paddle vertical position is connected to the robot's endpoint position along the $\mathrm{z}$ axis (Fig. 2). The robot is operated by the human thanks to a handle connected to its end-effector with an interface including a force/torque ( $\mathrm{f} / \mathrm{t}$ ) sensor (see Section IV-A for further details). A scaling factor can be applied between real robot movement and the simulated environment to adjust the simulated kinematics.

As shown in Fig. 2, the simulated environment projected on a screen comprises: i) a paddle, only moving along the vertical axis, ii) a ball, also locked in a 1 DOF movement, iii) a target height $h$, (red dashed line). The goal is to reach a human cyclic behaviour, while bouncing the ball at a target 
height in the simulated environment. Since the considered bouncing task is uni-dimensional, a planar 3 DOF robot evolving in the vertical plane $(\mathcal{O} \boldsymbol{x} \boldsymbol{z})$ is sufficient for the task.

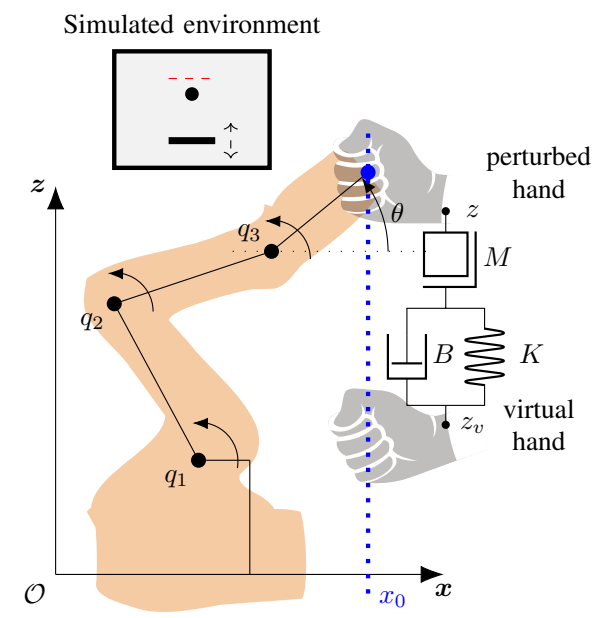

Fig. 2: Proposed interactive system for impedance estimation: a human interacts with the 3 DOF robot thanks to a handle. $q_{i}$ are the joint positions of the robot, $\theta$ is the endpoint orientation, $z$ is the endpoint position along the $\boldsymbol{z}$ axis, represented here after a perturbation responsible for the hand deviation from it's nominal trajectory denoted by $z_{v}$, the virtual position.

\section{B. Interactive robot control}

The complete control scheme is shown in Fig. 3. The robot block represents the robot arm dynamics with its inner joint currents and velocities loops. The control inputs to the robot are therefore joint reference velocities $\dot{q}_{d}$. The represented controller is composed of three control loops described below.

a) Main control loop: With respect to the ball bouncing task, the robot's apparent dynamics should be minimized to be as transparent as possible to the user. To this end, the main control loop realizes a Cartesian admittance control along the $\boldsymbol{z}$-axis based on the interaction forces $\boldsymbol{f}_{e}$, following a similar methodology to [17], [18]. Namely, $C_{z}$ is a single-input singleoutput (SISO) proportional-integral (PI) controller with gains $K_{p}$ and $K_{i}$. Note that the inverse Jacobian $\hat{\mathcal{J}}(q)^{-1} \in \mathbb{R}^{3 \times 3}$, is well defined in the particular case considered here, since the robot is maintained outside it's singular configurations by a secondary joint-space position controller. The tuning of the admittance control parameters is presented in Section IV-B.

b) Secondary control loop about $x_{0}$ : Since the studied ball bouncing task only occurs on the $z$-axis, the robot motion along the $\boldsymbol{x}$-axis should be minimized. To avoid the coupling effects in Cartesian space as much as possible, the main admittance controlled is realized at the endpoint level in the Cartesian space, only using the force along the $z$-axis. However, errors between the estimated and real Jacobian, as well as tracking errors in the velocity loops may generate small but undesired movements along the $\boldsymbol{x}$-axis. To alleviate this effect, an additional control loop is used to maintain the robot endpoint position about a constant $x_{0}$ position, using a Cartesian proportional control of gain $K_{x}$.

c) Secondary control loop about $q_{0}$ : A last sub-task is implemented to avoid unforeseen joint reversal and provide the robot a more predictable movement. The related secondary controller is therefore a simple joint position proportional control of gain $K_{2}$ about a given configuration $q_{0}$, which generates an additional joint speed reference $\dot{\boldsymbol{q}}_{2}$. Since this task should not interfere with the main one along the $z$ axis, it is projected to the main task's null space using the methodology described in [19], [20], and exploiting the fact that a 3 DOF manipulator is redundant for the realization of the main task. The Jacobian of the main task $\mathcal{J}_{1}$ is equivalent to the second row of the global Jacobian $\mathcal{J} \in \mathbb{R}^{3 \times 3}$. The projected joint speed reference $\dot{q}_{2}^{p}$ obtained by projection (5), is designed to have no effect on the $\boldsymbol{z}$-axis motion.

$$
\begin{aligned}
\dot{\boldsymbol{q}}_{2}^{p}= & \boldsymbol{N}_{2}(q) \dot{\boldsymbol{q}}_{2}=\boldsymbol{N}_{2}(q) K_{2}\left(\boldsymbol{q}_{0}-\boldsymbol{q}\right) \\
& \boldsymbol{N}_{2}(q)=\boldsymbol{I}-\mathcal{J}_{\mathbf{1}}(q)^{\boldsymbol{I}+} \mathcal{J}_{\mathbf{1}}(q)
\end{aligned}
$$

where the pseudo-inverse is defined by $A^{I+}=$ $\boldsymbol{I}^{-1} \boldsymbol{A}^{t}\left(\boldsymbol{A} \boldsymbol{I}^{-1} \boldsymbol{A}^{t}\right)^{-1}$ with $\boldsymbol{I} \in \mathbb{R}^{3 \times 3}$ the identity matrix.

These three combined controllers provide the robot arm with the behaviour of a mass sliding along a vertical line.

\section{Human impedance estimation}

1) Perturbation inputs: To estimate the arm endpoint impedance of a human during a co-manipulation, as described in Section II-B, perturbations need to be introduced in the task. To obtain brief perturbations $(<50 \mathrm{~ms})$, the inner velocity control loop is bypassed and the robot is torquecontrolled during the perturbation window. In this time frame, the outer-loop control of the robot is considered constant, since it has a response time longer than $50 \mathrm{~ms}$. Therefore, the last torque input $\tau_{0}$ before the perturbation is added to the perturbation torque corresponding to an endpoint force $\boldsymbol{f}_{p}$ and transformed to the joint level using the transposed Jacobian. The resulting input torque for the robot inner loop is given by $\boldsymbol{\tau}=\mathcal{J}(q)^{t} \boldsymbol{f}_{p}+\boldsymbol{\tau}_{0}$.

For the considered task, $\boldsymbol{f}_{p}$ has only a component along the $z$-axis. For the impedance identification, these perturbations are introduced at pseudo-random instants of the paddle cycle. In future experiments, the instants of the perturbation will be more finely controlled, but still need to have some randomness to prevent the participants to anticipate the perturbations. Their magnitude is constant in a trial, but their sign is random.

2) Virtual trajectory and force: The considered impedance estimation method is based on the fact that the perturbations induce deviations $\delta \boldsymbol{f}$ and $\delta \boldsymbol{x}$ (cf (3)) from the virtual trajectories, which would have taken place without perturbations. By essence unknown, these virtual trajectories both in hand positions and forces need to be reconstructed. In the literature, the average previous unperturbed trajectories were used in [25], while [26] fitted the cyclic patterns of previous data to a sinusoidal expression. A prediction algorithm with an unbiased error estimation, lesser than $1 \mathrm{~cm}$ after $200 \mathrm{~ms}$, was proposed in [16]. Since the impedance evaluation is done offline, the trajectory of the arm after the perturbation is available. Looking at the arm unperturbed trajectory given in Fig. 6, it can be observed that the movement can be approached using cubic splines. Fig. 6 presents an example of an estimated virtual trajectory against a real perturbed trajectory. More details about this spline-based reconstruction and it's influence on the results are given in Section V-B. 


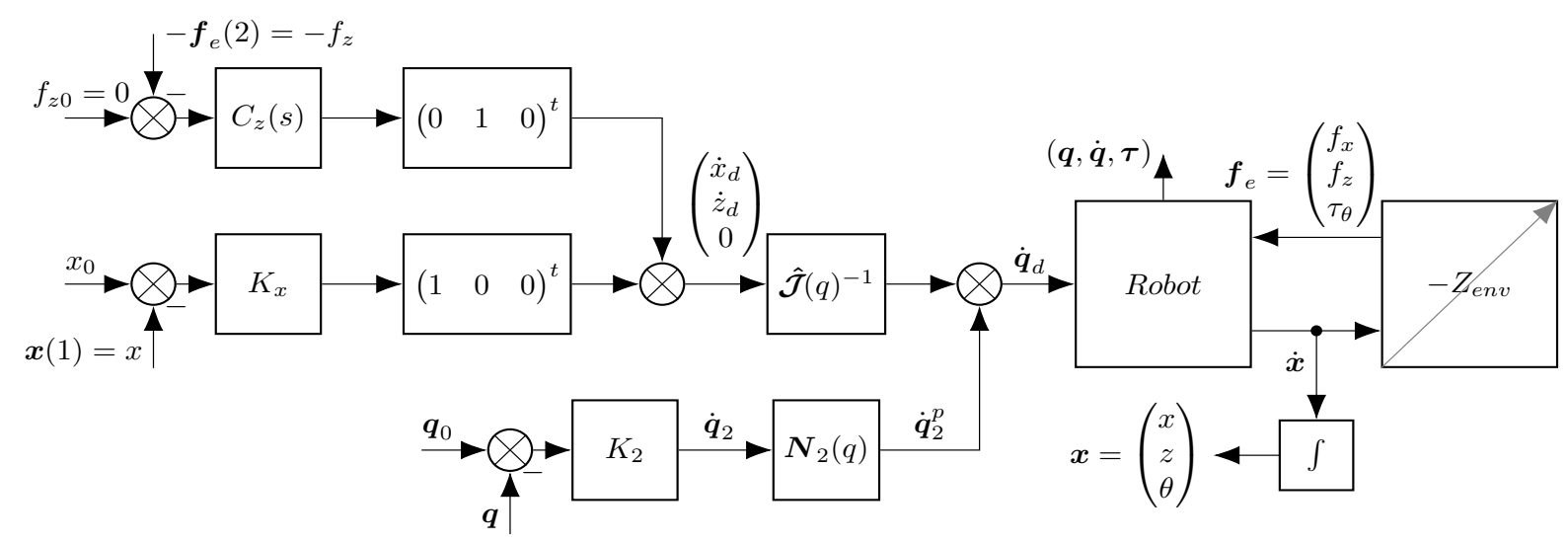

Fig. 3: Block diagram of the interactive robot control for a guided task on a 1 dimensional trajectory along the $\boldsymbol{z}$ axis. $\boldsymbol{f}_{e}$ represents the interaction forces in the robot base frame, $\boldsymbol{q}$ the robot joint positions, $\boldsymbol{\tau}$ the joint torques and $\boldsymbol{x}$ the robot's endpoint Cartesian coordinates. $C_{z}=K_{p}+\frac{K_{i}}{s}$ is a proportional integral (PI) controller responsible for the interactive admittance control.

3) Parameters estimation: Based on (4), the impedance parameter vector $\boldsymbol{\xi}=\left[\begin{array}{lll}K_{z} & B_{z} & I_{z}\end{array}\right]^{T}$ can be estimated by linear mean squares, solving the overdetermined system of equations resulting from the evaluation of (7) on a perturbed trajectory. Residuals are denoted $\rho$.

$$
\delta f_{z}=\left[\begin{array}{lll}
\delta x_{z} & \delta \dot{x}_{z} & \delta \ddot{x}_{z}
\end{array}\right] \boldsymbol{\xi}+\rho
$$

The identification being performed offline, centered differences can be used to obtain $\delta \dot{x}_{z}$ and $\delta \ddot{x}_{z}$.

\section{EXPERIMENTAL IMPLEMENTATION}

This section details the specific hardware and software choices that were made for the implementation of the methodology presented in Section III.

\section{A. Apparatus}

The robot used in this study is the KUKA ${ }^{\mathrm{TM}}$ youBot arm. Low-level joint current and velocity PI control loops are embedded into the motor servo controller modules, with control gains adjusted compared to the factory settings. Higher level control loops (position, force) are implemented using ROS. A rate of approximately $1 \mathrm{kHz}$ for the higher level control loops is achieved. The first and the last joints of the 5 DOF robotic arm are not required for the experiment and are thus maintained at fixed positions through joint position control. On Fig. 2, $q_{1}, q_{2}, q_{3}$ refer to respectively the $2^{\text {nd }}, 3^{\text {rd }}$ and $4^{\text {th }}$ joints of the robotic arm.

External sensors integrated to this setup are the ATI ${ }^{\mathrm{TM}}$ Mini45 6 axis f/t sensor and the Optitrack V120:Trio motion capture camera (mocap). Measurements from the $\mathrm{f} / \mathrm{t}$ sensor are acquired at $1 \mathrm{kHz}$, and are used in real time for the admittance control loop (Fig. 2). The camera measurements have a rate of $120 \mathrm{~Hz}$ and are used in this study only for offline analysis purposes. The acquired mocap position corresponds to the robot handle position held by the human hand.

To compute the interaction forces used in Fig. 3, the $\mathrm{f} / \mathrm{t}$ sensor measurements are debiased and transformed into the robot base frame, and the effect of gravity on the sensor is compensated. The obtained interaction forces are low-pass filtered at $30 \mathrm{~Hz}$ using a $4^{\text {th }}$ order Butterworth digital filter, and only the vertical force component $f_{z}$ is used in closedloop.

For the offline analysis and impedance estimation, unfiltered interaction forces are considered and post-treated differently from the control loop. Force and mocap positions are interpolated at $1 \mathrm{kHz}$ and low-pass filtered at $25 \mathrm{~Hz}$ using the same zero-phase fourth order Butterworth filter. These post-treated forces and mocap positions correspond to the notations $f_{z}$ and $x_{z}$ in (4).

\section{B. Admittance control gains tuning}

The admittance control gains are subject to the classical performance-robustness trade-off. To improve transparency and reduce the apparent robot dynamics, higher gains are desired. At the same time, considering that large scale experiments involve several participants, the admittance control loop need to be robust enough to remain stable when in contact with a large range of stiffnesses (i.e. participants hand/arm co-contractions), which limits the control gains. It is known that a robot inertia cannot be completely compensated, and its reduction is limited by the stability [18].

To ensure safe and robust co-manipulation, a stability map was experimentally determined, under two different conditions. The first condition corresponds to a manipulation performed under the worst possible environment, that is, moving the robot in rhythm with intentionally strong co-contraction of both the human hand and arm. The second condition corresponds to a nominal ball bouncing manipulation with lower co-contractions of the arm. In each condition, the resulting closed-loop behavior was sorted according to the following four categories: i) Unstable, impracticable, ii) Critical, barely practicable, iii) Badly damped, practicable but uncomfortable, iv) Well damped, or with no oscillation, comfortable. Conceptual behavior of the closed-loop signals is shown on Fig. 4b. The results of the stability map are displayed in Fig. 4a, the shaped bullets indicating the tested $\left(K_{p}, K_{i}\right)$ pairs for the first condition, and the green hatched area indicating the comfortable (iv) category for the second condition among which the gains used in experiments were selected. The comfortable stability region highlighted in the second test condition is larger that the co-contracted 
test condition as it can be expected. More detailed stability analysis is planned in future work.

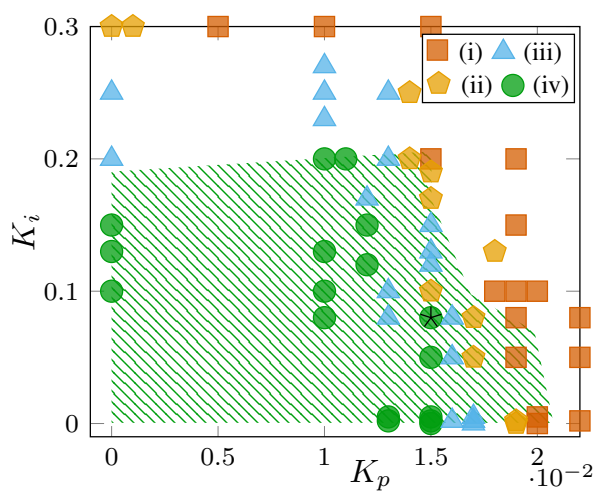

(a)

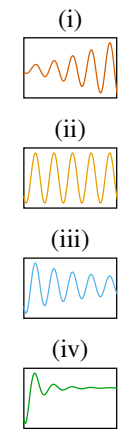

(b)
Fig. 4: (a) Experimental stability mapping depending on the admittance control gains. Condition 1: co-manipulation with intentionally high cocontractions of both hand and arm (shapes). Condition 2: co-manipulation with normal co-contractions of both hand and arm during ball bouncing (green hatched area for comfortable manipulation (iv)). The gains used in the experimental set up are indicated with a star. (b) Categories of typical force responses.

\section{FIRST EXPERIMENTAL RESULTS}

This section presents preliminary results demonstrating the feasibility of the task under the proposed protocol and first insights into impedance identification. More measurements need to be conducted for consolidated and statistically significant results. Future experiments are planned to include several expert participants to achieve longer stable rhythmic movements, with randomized but controlled timing of the perturbations. A calibration with a known mass-spring-damper system is also to be included.

\section{A. Feasibility of the ball bouncing task}

The domain of validity of the task was presented in Section II-A.2. It has been demonstrated feasible with a robot, through two experiments, considering the conditions given in Table I.

\begin{tabular}{|c|c|c|c|c|c|}
\hline$f(\mathrm{~Hz})$ & $h(\mathrm{~m})^{\mathrm{a}}$ & $g\left(\mathrm{~m} \mathrm{~s}^{-2}\right)$ & $\dot{z}_{k}^{+}\left(\mathrm{m} \mathrm{s}^{-1}\right)^{\mathrm{a}}$ & $\alpha$ & $z_{k}(\mathrm{~m})^{\mathrm{a}}$ \\
\hline$[0.9 ; 1.7]$ & {$[0.8 ; 1.0]$} & 9.81 & {$[1.32 ; 3.34]$} & 0.6 & 0 \\
\hline
\end{tabular}
a In the simulated environment.

TABLE I: Tested conditions

Fig. 5 shows a part of a bouncing session with perturbations, during a co-manipulation with the robot. The experiment was conducted by the authors themselves. A cyclic behaviour is effectively obtained. It is important to underline that a novice participant needs to perform training experiments to be able to reach the expert level. Participants already practicing juggling tasks are more comfortable with the proposed protocol.

\section{B. Impedance estimation - first results}

1) Time window selection for estimation: As mentioned in [22], and reminded in Section II-B, voluntary reactions need to be discarded, because they cannot be properly modeled by (3). Voluntary reactions response time is typically of $100 \mathrm{~ms}$. Yet, at a $1 \mathrm{kHz}$ rate, [22] found that using only $100 \mathrm{~ms}$ of data after the perturbation did not bring satisfying results. Therefore,

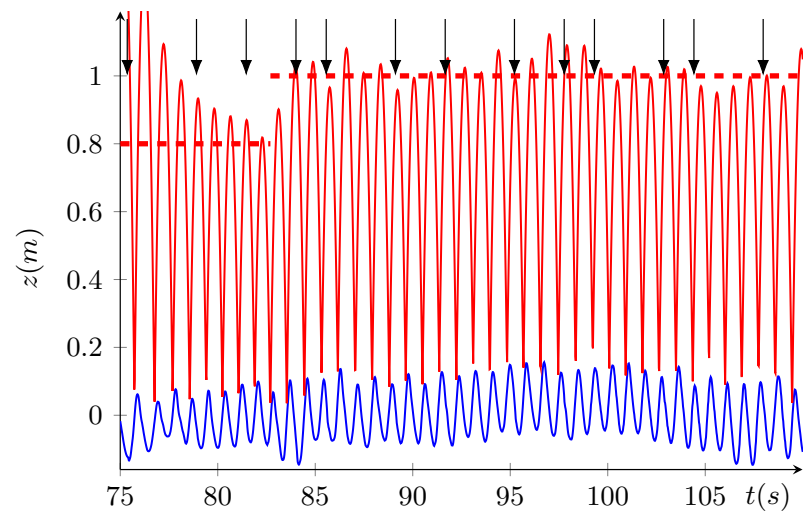

Fig. 5: Ball bouncing task in the simulated environment. The paddle vertical position (solid blue line) reflects the collaborative robot endpoint position. The ball position (solid red line), and the target height (dashed red line) are depicted. All the data are expressed in the coordinates of the simulated environment. Perturbation timings are indicated with arrows.

they assumed that voluntary actions were not dominant in the first $200 \mathrm{~ms}$, and used that time window for the evaluation. In the present work, a time window $t_{\text {estim }}$ of $200 \mathrm{~ms}$ was selected for the estimation, based on the literature, and on the direct observation that this time window concentrated the main perturbation effects during the task (see Fig. 6).

In these preliminary experiments, perturbations were induced at randomly chosen instants. For meaningful comparisons and averaging of the estimated parameters, only $n_{p}=11$ perturbations not too close to paddle position peaks are selected in the presented results.

2) Virtual trajectories parameters selection: The virtual trajectories were obtained using cubic spline interpolation in MATLAB. The interpolation was done at $1 \mathrm{kHz}$, to estimate the virtual trajectory on $t_{\text {estim }}$ after a perturbation onset $t_{p}$, using data immediately before and after the interval of interest to be reconstructed. To evaluate the quality of this reconstruction, it was applied both on perturbed and non-perturbed cycles (see Fig. 6 for an example). An additional parameter, a delay $\delta t$ between the beginning of the reconstructed interval with respect to $t_{p}$ was introduced for comparison purposes. This delay parameter was found to have non negligible influence on the parameter estimation results. Especially, for $0 \leq \delta t \leq 5 \mathrm{~ms}$, negative parameters were obtained with lower determination coefficient $R^{2}(\leq 0.9)$ for the linear fit. Incrementally increasing $\delta t$ to $20 \mathrm{~ms}$ shows, for the perturbed intervals, a convergence of the mean estimated parameters to constant mean values with significantly reduced relative standard deviations and a dramatically improved mean $R^{2}$ of 0.96 , against no significant changes on non-perturbed intervals where $R^{2}$ remains smaller that 0.6.

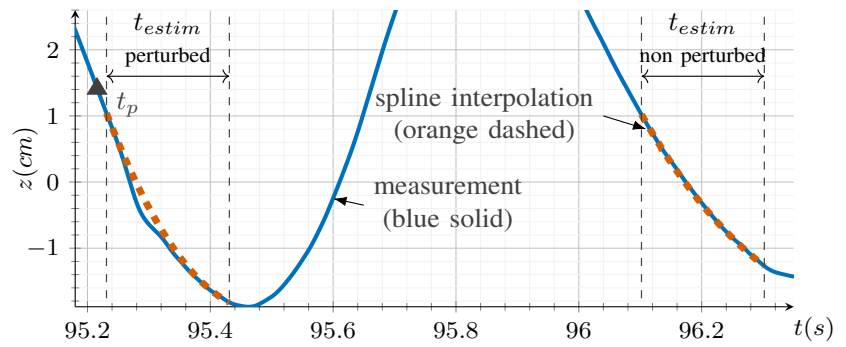

Fig. 6: Position trajectory reconstruction, with and without perturbation. 

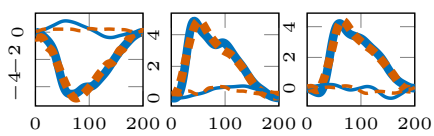

$\underbrace{1}_{0}$

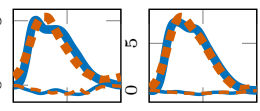

$\stackrel{0}{i}+1$

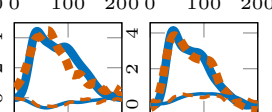

$\begin{array}{lllllll}0 & 100 & 2000 & 100 & 200 & 100 & 200\end{array}$

Fig. 7: Force deviations $\delta f_{z}(\mathrm{~N})$ from the virtual trajectory: comparison between perturbed (bold lines) and non-perturbed (thin lines) cycles, on $200 \mathrm{~ms}$ intervals. $\delta f_{z}$ used in identification (solid blue lines) are compared to the reconstructions $\delta \hat{f}_{z}$ (orange dashed lines) based on the identified impedance parameters.

3) Parameters estimation: In [3], [22]-[24] endpoint parameters for the stiffness were found with a magnitude ranging between 40 and $700 \mathrm{~N} \mathrm{~m}^{-1}$, under static and moving configurations, with and without load for the human arm. Preliminary results from the authors' trials show most promising results using $200 \mathrm{~ms}$ for the impedance identification, with $20 \geq \delta t \geq 10 \mathrm{~ms}$, possibly to abide by latency in the system. The stiffnesses $K_{z}$ found are higher than those mentioned above, with an average of $1140 \mathrm{~N} \mathrm{~m}^{-1}$ and relative standard deviation of $27 \%$ on the $n_{p}$ perturbed intervals. Further experiments are to be conducted. Reconstructed forces using the identified parameters seem to explain most of the relation between force and movement on perturbed intervals (Fig. 7).

\section{CONCLUSIONS}

The presented first results demonstrate the feasibility of using interactive robotics for human impedance estimation. Several aspects of the proposed procedure can however be improved. The use of cubic splines for trajectory estimation yields acceptable results for both the positions and velocities, but since cubic splines are $C^{2}$, poorly estimated accelerations were obtained. Using similar trajectory reconstruction techniques as those described in [16] could improve both virtual trajectory and force estimations. Other techniques than the presented least squares could be considered to improve the KBI model identification, for example based on linear dynamic system identification methods.

Finally, larger-scale data acquisition on a statistically significant number of experiments with several participants is expected to provide more in-depth insights on the possible cyclic variations of the human endpoint impedance, during a task with physical interaction. Understanding these variations could be used for bio-mimetic rhythmic control design for collaborative robots in interaction with their environment.

\section{REFERENCES}

[1] R. J. Jagacinski and J. M. Flach, 'Control theory for humans: Quantitative approaches to modeling performance', Mahwah, NJ: Erlbaum, 2003.

[2] M.L. Latash, et al., 'Motor control theories and their applications', Medicina 2010, 46, 382

[3] T. Tsuji and Y. Tanaka, 'Bio-mimetic impedance control of robotic manipulator for dynamic contact tasks', Robotics and Autonomous Systems, vol. 56, no. 4, pp. 306-316, 2008.
[4] E. Burdet, et al., 'The central nervous system stabilizes unstable dynamics by learning optimal impedance', Nature, vol. 414, no. 6862, pp. 446-449, 2001.

[5] J. Zawadzki and A. Siemieński, 'Maximal frequency, amplitude, kinetic energy and elbow joint stiffness in cyclic movements', Acta Bioeng Biomech, vol. 12, no. 2, pp. 55-64, 2010.

[6] Y. Z. Arslan, et al., 'Exoskeletons, Exomusculatures, Exosuits: Dynamic Modeling and Simulation', Biomechatronics, Elsevier, 2019, pp. 305331 .

[7] N. Hogan, 'Adaptive control of mechanical impedance by coactivation of antagonist muscles', IEEE Trans. Autom. Control, vol. 29, no. 8, pp. 681-690,1984.

[8] D. Formica, et al., 'A geometric framework for the estimation of joint stiffness of the human wrist*', 16th IEEE Int Conf Rehabil Robot, 2019, pp. 151-156.

[9] P. Maurice, V. et al., 'Chapter 60 - Digital human modeling for collaborative robotics', in DHM and Posturography, S. Scataglini and G. Paul, Eds. Academic Press, 2019, pp. 771-779.

[10] D. Piovesan, et al., 'Measuring multi-joint stiffness during single movements: numerical validation of a novel time-frequency approach.', PLoS One, vol. 7, no. 3, pp. e33086-e33086, 2012.

[11] N. Hogan and D. Sternad, 'On rhythmic and discrete movements: reflections, definitions and implications for motor control', Exp Brain Res, vol. 181, no. 1, pp. 13-30, 2007.

[12] Y. Huang and Q. Wang, 'Torque-Stiffness-Controlled Dynamic Walking: Analysis of the Behaviors of Bipeds with Both Adaptable Joint Torque and Joint Stiffness', IEEE Robot Autom Mag, vol. 23, no. 1, pp. 71-82, 2016.

[13] G. Avrin, et al., 'The self-organization of ball bouncing', Biol. Cybern. (Modeling), Springer Verlag, 2018.

[14] I. A. Siegler, B. G.Bardy, and W. H. Warren, 'Passive vs. active control of rhythmic ball bouncing: The role of visual information', J. Exp. Psychol. Hum. Percept. Perform., 36(3), 729-750, 2010.

[15] T. Tsuji, 'Human Arm Impedance in Multi-Joint Movements', in Advances in Psychology, vol. 119, P. Morasso and V. Sanguineti, Eds. North-Holland, 1997, pp. 357-381.

[16] E. Burdet, et al., 'A method for measuring endpoint stiffness during multi-joint arm movements', J. Biomech., vol. 33, no. 12, pp. 17051709, 2000.

[17] W. S. Newman, 'Stability and Performance Limits of Interaction Controllers', J. Dyn. Sys., Meas., Control, vol. 114, no. 4, pp. 563-570, 1992.

[18] X. Lamy, et al., 'Achieving efficient and stable comanipulation through adaptation to changes in human arm impedance', IEEE ICRA, 2009, pp. 265-271.

[19] A. Dietrich, C. Ott, and A. Albu-Schäffer, 'An overview of null space projections for redundant, torque-controlled robots', Int. J. Robot. Res., vol. 34, no. 11, pp. 1385-1400, 2015

[20] H. Sadeghian, et al., 'Multi-priority control in redundant robotic systems', IEEE/RSJ IROS, pp. 3752-3757, 2011.

[21] D. L. Guarin and R. E. Kearney, 'Unbiased Estimation of Human Joint Intrinsic Mechanical Properties During Movement', IEEE Trans. Neural Syst. Rehabil. Eng., vol. 26, no. 10, pp. 1975-1984, 2018.

[22] M. S. Erden and A. Billard, 'End-Point Impedance Measurements Across Dominant and Nondominant Hands and Robotic Assistance with Directional Damping', IEEE Trans. Cybern., vol. 45, no. 6, pp. 1146-1157, 2015.

[23] J. M. Dolan, M. B. Friedman, and M. L. Nagurka, 'Dynamic and loaded impedance components in the maintenance of human arm posture', IEEE Trans. Syst., Man, Cybern. Syst., vol. 23, no. 3, pp. 698-709, 1993.

[24] F. A. Mussa-Ivaldi, N. Hogan, and E. Bizzi, 'Neural, mechanical, and geometric factors subserving arm posture in humans', J. Neurosci., vol. 5, no. 10 , pp. 2732-2743, 1985

[25] D. J. Bennett, et al., 'Time-varying stiffness of human elbow joint during cyclic voluntary movement', Exp Brain Res, vol. 88, no. 2, pp. 433-442, 1992.

[26] M. O. Abe and N. Yamada, 'Modulation of elbow joint stiffness in a vertical plane during cyclic movement at lower or higher frequencies than natural frequency', Exp Brain Res, vol. 153, no. 3, pp. 394-399, 2003.

[27] G. Avrin, et al., 'Dynamic Stability of Repeated Agent-Environment Interactions During the Hybrid Ball-bouncing Task', ICINCO, pp. 486-496, 2017 